\title{
Heterologous Expression of ZmNF-YA12 Confers Tolerance to Drought and Salt Stress in Arabidopsis
}

\section{Tongtong Zhang}

Capital Normal University

Dengyu Zheng

Beijing Academy of Agriculture and Forestry Sciences

Chun Zhang

Beijing Academy of Agriculture and Forestry Sciences

Zhongyi Wu

Beijing Academy of Agriculture and Forestry Sciences

\section{Rong Yu}

Capital Normal University

Zhongbao Zhang ( $\sim$ happyzzb@126.com )

Beijing Academy of Agriculture and Forestry Sciences https://orcid.org/0000-0001-5494-9762

\section{Research Article}

Keywords: Maize, drought tolerance, salt tolerance, NF-YA, Arabidopsis

Posted Date: December 9th, 2021

DOI: https://doi.org/10.21203/rs.3.rs-1010623/v1

License: (c) (i) This work is licensed under a Creative Commons Attribution 4.0 International License.

Read Full License 


\section{Abstract}

Drought and salinity are serious environmental factors limiting the growth and productivity of plants worldwide. Therefore, it is necessary to develop ways to improve drought and salinity stress tolerance in plants. In this study, a drought-responsive nuclear factor $Y$ subunit A gene, ZmNF-YA12, was cloned from maize. qPCR revealed $Z m N F-Y A 12$ transcript in all vegetative and reproductive tissues, with higher levels in young roots. Expression analyses of maize revealed that ZmNF-YA12 was induced by abscisic acid $(A B A)$, jasmonic acid (JA), and abiotic stresses, including dehydration, high salinity, cold, and polyethylene glycol (PEG) treatment. The heterologous expression of ZmNF-YA12 in Arabidopsis plants resulted in increased root length and better plant growth than in wild-type (WT) plants under conditions of mannitol, salt, and JA stress on 1/2 MS medium. Transgenic Arabidopsis showed improved tolerance to drought and salt stresses in soil, and higher proline content and lower malondialdehyde (MDA) content than WT controls. The transgenic plants also maintained higher peroxidase (POD) activities than WT plants under conditions of $\mathrm{NaCl}$ stress. A yeast two-hybrid experiment demonstrated that ZmNF-YA12 interacted with ZmNF-YC1 and ZmNF-YC15. Moreover, the transcript levels of stress-responsive genes ( $R D 29 A, R D 29 B, R A B 18$, and $R D 22$ ) were markedly increased in transgenic lines under conditions of drought and salt stress. These observations suggested that the ZmNF-YA12 gene confers drought and salt stress tolerance, and has potential applications in molecular breeding with maintenance of production under conditions of stress.

\section{Introduction}

The crop yield of maize (Zea mays L.), the most widely grown cereal crop in the world, is considerably limited by a range of abiotic stress factors (Pechanova et al. 2013). Drought and increased soil salinity are projected to lead to a $50 \%$ loss of arable land by the year 2050 , which will severely affect crop yields. Therefore, it is becoming increasingly important to improve water use efficiency and salt tolerance for agricultural production in the ever-decreasing area of arable land (Deinlein et al. 2014; Tiburcio et al. 2012). Transcription factors play important roles in abiotic stress responses in plants, and represent promising targets for the genetic engineering of plants with elevated stress resistance (Nowicka et al. 2018). Many plant genes are regulated in response to abiotic stresses, and the products of these genes have functions related to the stress responses and tolerance (Yamaguchi-Shinozaki and Shinozaki 2006).

Nuclear factor $Y$ (NF-Y), also called CBF and CP1, consists of three different subunits (NF-YA, NF-YB, and NF-YC), and is a unique DNA-binding protein that interacts with the CCAAT motif, a common element present in the promoters of a number of mammalian genes (Maity and de Crombrugghe 1998). The core domains of the NF-YC/NF-YB proteins interact through histone fold motifs. This histone-like pair is closely related to the $\mathrm{H} 2 \mathrm{~A} / \mathrm{H} 2 \mathrm{~B}$ and $\mathrm{NC} 2 \alpha / \mathrm{NC} 2 \beta$ families, both of which have features common to this class of proteins and unique to NF-Y (Romier et al. 2003).

The plant NF-Y transcription factors have been reported to be key players in plant-microbe interactions, root development, and stress tolerance. Some members of the NF-YA and NF-YB gene families have been 
shown to be involved in responses to water and nutrient scarcity in mono- and dicotyledonous plants (Zanetti et al. 2017). AtNF-YB2 and AtNF-YB3 are both essential for the normal flowering induced by long days in Arabidopsis (Kumimoto et al. 2008). Transcription of OsNF-YA7 was shown to be induced by drought stress, and its overexpression in transgenic rice plants enhanced their drought tolerance (Lee et al. 2015). Overexpression of TaNF-YA10-1 in wheat conferred drought tolerance, with longer root length and better whole-plant growth under conditions of drought (Ma et al. 2015). Transgenic tobacco plants overexpressing CSNF-YA5 showed superior growth and photosynthetic rates under both normal conditions and drought stress (Pereira et al. 2018). PdNF-YB21 overexpression promoted root growth with highly lignified and enlarged xylem vessels in poplar, resulting in increased drought resistance (Zhou et al. 2020). However, the biological roles of many members of the NF-Y family in maize are not clear.

Previously, we reported that ZmNF-YA12 (GRMZM5G857944) could respond to abiotic stress (Zhang et al. 2016). To investigate the molecular biology function of $Z m N F-Y A 12$, its tissue-specific expression, gene expression patterns under different exogenous stresses, heterologous expression in Arabidopsis, and yeast two-hybrid experiment were performed. Transgenic Arabidopsis lines expressing ZmNF-YA12 showed improved drought and NaCl tolerance.ZmNF-YA12 interacts with ZmNF-YC1 and ZmNF-YC15. Our data suggest that $Z m N F-Y A 12$ may represent an important mechanism underlying the function of NF$\mathrm{Y}$ under $\mathrm{NaCl}$ and drought stress.

\section{Materials And Methods}

\section{Isolation of ZmNF-YA12}

Total RNA was isolated from maize seedlings using TRIzol reagent (CW Biotech, Beijing, China) and reverse-transcribed to complementary DNA (cDNA) using HiScript II Q RT SuperMIX (Vazyme, Nanjing, China). The full ZmNF-YA12cDNA was amplified by PCR using the forward primer (FP) 5'ATGCTTCTTCCCTCTTCGTCTT-3' and reserve primer (RP) 5'-TCATCTCATAACTGGAACCCT-3'.

\section{Plant materials, growth conditions, and treatments}

For tissue-specific analysis, leaves, stems, and roots were harvested from three-leaf seedlings grown in a greenhouse $\left(28^{\circ} \mathrm{C}, 16 / 8 \mathrm{~h}\right.$ day/night cycle). Mature leaves, roots, silks, tassels, and embryos were harvested at the grain-filling stage from plants grown in the field. All harvested materials were frozen immediately in liquid nitrogen and stored at $-80^{\circ} \mathrm{C}$.

To determine the ZmNF-YA12 expression patterns under various stress conditions, maize seedlings were grown in 12-cm hydroponic barrels containing nutrient solution. Three-leaf maize seedlings were subjected to dehydration, $\mathrm{NaCl}$, polyethylene glycol (PEG), cold, abscisic acid (ABA), and jasmonic acid (JA) treatments. For dehydration treatment, the whole seedling was removed, washed, and placed on an experimental table for natural dehydration at room temperature $\left(25^{\circ} \mathrm{C}\right)$. For salt and PEG treatments, the roots of the seedlings were immersed in solutions containing $200 \mathrm{mM} \mathrm{NaCl}$ and $20 \%$ PEG, respectively. For cold treatment, seedlings were kept at $4^{\circ} \mathrm{C}$. For each of the above four treatments, the shoots and 
roots were collected at $0,1,2,5,10$, and 24 hours after treatment. For the ABA and JA treatments, the leaves of the seedlings were sprayed with solutions containing $100 \mu \mathrm{M}$ ABA or $100 \mu \mathrm{M} \mathrm{JA}$ and covered with plastic film. The leaves were then collected at 0, 1, 2, 5, and 10 hours after treatment. The samples were immediately frozen with liquid nitrogen for isolation of RNA.

Arabidopsis thaliana ecotype Columbia (Col-0) was used for transformation in this study. After vernalization treatment, seeds were surface-sterilized in a solution of $0.5 \% \mathrm{NaClO}$ for 10 minutes, and washed five times with sterile distilled water. Following this treatment, the seeds were germinated and grown on half-strength Murashige and Skoog (1/2 MS) medium ( $\mathrm{pH} 5.8-6.0)$. The plates were transferred to a growth chamber at $22^{\circ} \mathrm{C}$ for germination.

\section{Gene expression analysis using qPCR}

cDNA samples were obtained as described above. qPCR was performed in a CFX Connect Real-time PCR system (Bio-Rad, Hercules, CA, USA) using a Super Real PreMix (SYBR Green) kit (Tsingke Biotech, Beijing, China) according to the manufacturer's instructions. The primers used for qPCR were designed according to the ZmNF-YA12 CDNA sequence (FP: 5'-AGCAACCTCCATTTGCGAGTCA-3' and RP: 5'-

GGCTGCCCAAACATCTCCTGAT-3'). Each reaction was performed in triplicate, and the results are expressed relative to the expression levels of Actin (FP: 5'-GGTAACATTGTGCTCAGTGGTGG-3' and RP: 5'AACGACCTTAATCTTCATGCTGC-3') and GAPDH (FP: 5'-CCCTTCATCACCACGGACTAC-3' and RP: 5'AACCTTCTTGGCACCACCCT-3') in each sample using the $2^{-\triangle A C T}$ method.

\section{Vector construction and transformation of Arabidopsis}

The ZmNF-YA12 cDNA was cloned into the pCAMBIA-3301 vector driven by the cauliflower mosaic virus (CaMV) 35S promoter (Supplementary Fig. 1), as confirmed by sequencing. The resulting construct was transformed into Arabidopsis Col-0 by the floral dip method using Agrobacterium tumefaciens GV3101. The first generation ( $T_{0}$ ) seeds of transgenic Arabidopsis were screened on 1/2 MS medium containing $50 \mathrm{mg} / \mathrm{L}$ kanamycin, and transgenic plants were confirmed by PCR and qPCR (FP: 5'AACTCATCTGCGGCTTGG-3' and RP: 5'-GTATAATTGCGGGACTCTAATC-3'; and FP: 5'AGCAACCTCCATTTGCGAGTCA -3' and RP: 5'-GGCTGCCCAAACATCTCCTGAT -3', respectively). Homozygous plants of the $T_{3}$ generation were used for further analysis.

\section{Root growth assay}

For root growth assay, transgenic and wild-type (WT) seeds were placed on 1/2 MS agar plates for germination. Seven days later, five germinated seedlings of the same size from each line were carefully transferred to 1/2 MS agar plates supplemented with $150 \mathrm{mM} \mathrm{NaCl}, 150 \mathrm{mM}$ mannitol, $50 \mu \mathrm{M} \mathrm{JA}$, or 10 $\mu \mathrm{M}$ ABA. Seedling root lengths were measured using Image J software ( $\mathrm{NIH}$, Bethesda, MD, USA) after 8 days of upright growth in treatment medium.

\section{Drought and $\mathrm{NaCl}$ treatment of transgenic Arabidopsis}


Drought and $\mathrm{NaCl}$ tolerance assays were performed on seedlings grown in pots in a greenhouse. Transgenic and WT seeds were germinated on 1/2 MS medium. One-week-old seedlings were planted in 7-cm pots containing mixed soil (vermiculite: humus $=1: 1$ ) of equal quality and well-watered for 3 weeks. For drought stress treatment, the seedlings were subsequently cultured without watering for 3 weeks and then re-watered for 2 days. For $\mathrm{NaCl}$ stress treatment, the plants were irrigated with a solution containing $450 \mathrm{mM} \mathrm{NaCl}$ for 1 week. Drought and $\mathrm{NaCl}$ tolerance experiments were performed in triplicate.Samples of Arabidopsis leaves were collected after the seedlings exhibited distinct phenotypes under drought and salt treatments. The peroxidase (POD) activity, proline, malondialdehyde (MDA), and chlorophyll contents were measured using a commercial assay kit (Solarbio, Beijing, China) according to the manufacturer's instructions.

\section{Yeast two-hybrid assays}

For yeast two-hybrid analysis, the ZmNF-YA12 cDNA was cloned into the bait plasmid pGBKT7 (pGBKT7ZmNF-YA12). The full-length cDNAs of ZmNF-YB7 (GRMZM2G169884_T01), ZmNF-YC1

(GRMZM2G089812_T01), ZmNF-YC15(GRMZM2G124421_T01), ZmNF-YC17

(GRMZM2G311316_T01), ZmCOI1(GRMZM2G151536), and ZmMYC2 (GRMZM2G049229) were separately cloned into the target PGADT7 plasmid. The bait and target plasmids to be tested for interactions were co-transformed into the yeast strain AH109 and plated on synthetic defined (SD) medium lacking leucine and tryptophan (SD/-LT) for screening transformants. The independent transformed colonies were then grown on SD medium lacking leucine, tryptophan, adenine, and histidine (SD/-AHLT). Survival of the yeast colonies on SD/-AHLT medium indicated that the target gene can interact with ZmNF-YA12.

\section{Statistical analysis}

The experiments were repeated three times and the data are presented as the mean \pm SEM. The significance of the differences in the data was determined using SPSS statistical software (v. 25.0; SPSS Inc., Chicago, IL, USA). In all analyses, $p<0.05$ was taken to indicate statistical significance.

\section{Results}

\section{Isolation and characterization of ZmNF-YA12}

The full-length ZmNF-YA12 cDNA is 816 bp in length and encodes 271 amino acid residues with a predicted molecular mass of $29.3 \mathrm{kDa}$ and isoelectric point $(\mathrm{p} /$ ) of 10.96. Protein structure alignment showed that the ZmNF-YA12 sequence included an NF-Y transcription factor conserved domain (Fig. 1A). The results indicated that $Z m N F-Y A 12$ is a member of the NF-YA transcription factor family. The deduced amino acid sequence of $Z m N F-Y A 12$ was further compared to other NF-YA proteins from various organisms by phylogenetic analysis (Fig. 1B). The results indicated that ZmNF-YA12 is closely related to OsHAP2E. Analysis of the ZmNF-YA12 promoter using the PlantCARE database (http://bioinformatics.psb.ugent.be/webtools/plantcare/html/) revealed a series of light-related and 
hormone stress response elements, including CAT-box, G-box, and CGTCA motif (Supplementary Fig. 2). The results suggest that $Z m N F-Y A 12$ may play important roles in responses to environmental stresses and regulation of plant growth and development.

\section{Expression pattern of maize ZmNF-YA12}

The expression levels of $Z m N F-Y A 12$ in different tissues under various stresses were determined by qPCR. The results showed that $Z m N F-Y A 12$ was expressed at higher levels in young roots than in other tissues (Fig. 2), and the expression levels of ZmNF-YA12 in shoots and roots were upregulated by dehydration and PEG treatments (Fig. 3A, B). Under conditions of cold and $\mathrm{NaCl}$ treatment, the expression of ZmNF-YA12 was markedly induced in roots but not in shoots (Fig. 3C, D). As shown in Fig. 3E, the ZmNF-YA12 transcript level was downregulated at 1, 3, and 5 hours, and upregulated at 10 hours, with ABA treatment. With JA treatment, the expression of ZmNF-YA12 first decreased and then increased, peaking at 3 hours (Fig. 3F).

\section{Tolerance of transgenic Arabidopsis plants to salt, mannitol, JA, and ABA stress}

To assess the effects of ZmNF-YA12 in responses to abiotic stresses, ZmNF-YA12 transgenic Arabidopsis plants (L-1, L-2, L-3) and WT seedlings were grown on 1/2 MS medium with different treatments. WT and transgenic plants showed similar root lengths under normal and $10 \mu \mathrm{M}$ ABA conditions. However, the roots of transgenic lines were much longer than those of WT plants in the presence of $150 \mathrm{mM} \mathrm{NaCl}, 150$ mM mannitol, or $50 \mu \mathrm{M}$ JA (Fig. 4; Supplementary Figs. 3-6).

\section{Heterologous expression of ZmNF-YA12 confers enhanced drought and salt tolerance in Arabidopsis}

Under control conditions, both WT and ZmNF-YA12 transgenic plants exhibited a similarly normal growth phenotype. Drought and salt stress significantly inhibited the growth of WT plants, which exhibited more wilted and smaller leaves (Fig. 5A and Fig. 6A). However, ZmNF-YA12 transgenic Arabidopsis plants showed less wilted leaves and more green and larger leaves (Fig. 5B and Fig. 6B). For drought treatment, after re-watering for 2 days, the ZmNF-YA12 transgenic lines recovered more quickly, grew more green leaves and appeared to be healthier than WT plants (Fig. 5C).

To further characterize the function of ZmNF-YA12, we examined the MDA and proline contents under drought treatment, and POD and chlorophyll contents under salt stress in transgenic lines and WT plants. As shown in Fig. 5D, MDA content was lower in the transgenic plants than WT controls under drought stress. As expected, the proline content was much higher in the transgenic lines than in WT plants (Fig. $5 \mathrm{E})$. Under $\mathrm{NaCl}$ treatment, the activities of POD in the transgenic lines were much higher than in WT plants (Fig. 6C). However, there were no obvious differences in chlorophyll contents between WT and transgenic plants (Fig. 6D). These results suggested that the heterologous expression of ZmNF-YA12 improves drought and salt tolerance in Arabidopsis.

To determine the mechanism underlying the involvement of $Z m N F-Y A 12$ in the stress response, the expression levels of stress-related genes were analyzed by qPCR in ZmNF-YA12 transgenic and WT 
plants grown under normal, $\mathrm{NaCl}$, and drought conditions. As shown in Fig. 7, the expression levels of the stress-related genes $R D 29 A, R D 29 B, R A B 18$, and $R D 22$ in the transgenic lines were much higher than in WT plants under $\mathrm{NaCl}$ and drought conditions.

\section{ZmNF-YA12 interacts with ZmNF-YC1 and ZmNF-YC15}

To investigate the mechanisms underlying the involvement of ZmNF-YA12 in stress regulation, yeast twohybrid assay was performed to identify proteins interacting with ZmNF-YA12. The results showed that $Z m N F-Y A 12$ has no self-transcriptional activation activity. All of the transformed yeast cells grew well on $\mathrm{SD} /$-LT medium, and the yeast cells transformed with both pGBKT7-ZmNF-YA12 + pGADT7-ZmNF-YC1 and pGBKT7-ZmNF-YA12 + pGADT7-ZmNF-YC15 grew well on SD/-AHLT medium, indicating that ZmNFYA12interacts with ZmNF-YC1 and ZmNF-YC15(Fig. 8).

\section{Discussion}

The growth, development, and productivity of maize are seriously affected by abiotic stresses, such as drought, salinity, high and low temperatures, and by biotic stresses, such as fungi, viruses, and pests (Gong et al. 2014). The NF-Y transcription factors are important regulators of plant development and responses to environmental stress (Petroni et al. 2012). The maize genome includes $50 Z m N F-Y$ genes (14 ZmNF-YA, $18 Z m N F-Y B$, and $18 Z m N F-Y C$ ) (Zhang et al. 2016). In this study, we identified and characterized a gene, $Z m N F-Y A 12$, related to stress tolerance. $Z m N F-Y A 12$ transcript levels were significantly induced by dehydration, PEG, cold, $\mathrm{NaCl}, \mathrm{ABA}$, and JA treatments (Fig. 3).

Several studies have indicated that $N F-Y$ genes are involved in stress responses. OsHAP2E, a homolog of ZmNF-YA12, confers biotic and abiotic resistance, and increased photosynthesis and tiller numbers in rice (Alam et al. 2015). Overexpression of SiNF-YA1 in transgenic tobacco lines enhanced drought and salt tolerance (Feng et al. 2015). Transgenic Arabidopsis plants overexpressing AhNF-YC showed increased seedling sensitivity to $A B A$, and influenced the expression of several genes associated with secondary metabolism, development, and ABA-related responses (Palmeros-Suárez et al. 2015). Our data showed that transgenic Arabidopsisseedlings expressing ZmNF-YA12 had longer roots than WT plants when grown on $1 / 2 \mathrm{MS}$ medium under mannitol, $\mathrm{NaCl}$, and JA treatments (Fig. 4). Furthermore, seedlings of ZmNF-YA12 transgenic plants grown in soil under drought and high $\mathrm{NaCl}$ conditions showed enhanced tolerance in comparison to WT plants (Fig. 5A and Fig. 6A). Several physiological and biochemical factors, such as MDA, proline, chlorophyll contents, and POD activity, play essential roles in plant tolerance to abiotic stresses. Proline plays a role as a compatible solute under conditions of environmental stress and contributes to the redox balance of the cell (Lehmann et al. 2010). MDA is the most frequently measured biomarker of oxidative stress, i.e., lipid peroxidation (Tsikas 2017). With drought treatment, transgenic ZmNF-YA12 plants showed lower MDA content and higher proline content than WT controls (Fig. 5D, 5E). Therefore, we concluded that ZmNF-YA12 transgenicplants have enhanced drought tolerance. Peroxiredoxins are thiol PODs with a variety of functions in the oxidation resistance and redox signaling networks of the cell (Liebthal et al. 2018). Leaf chlorophyll content 
represents the photosynthetic capacity, and high oxidative stress inhibits its synthesis and accumulation (Agathokleous et al. 2020). The levels of POD activity in 35S:ZmNF-YA12 Arabidopsis were much higher than in WT plants under high $\mathrm{NaCl}$ conditions (Fig. 6C). However, there were no distinct differences in chlorophyll content between the transgenic and WT plants (Fig. 6D). These results indicated that ZmNFYA12 has a positive effect on salinity, osmotic, and drought stress responses in plants.

To investigate the mechanisms of action of ZmNF-YA12 in stress responses, we examined the expression levels of stress-responsive genes. Previous studies showed that RD29A and RD29B can be induced by drought and salt stress, and responded to dehydration and ABA treatments (Msanne et al. 2011;

Nakashima et al. 2006). In addition, $R D 22$ and $R A B 18$ are marker genes for ABA-induced gene expression and key nodes in ABA-responsive signaling networks (Rushton et al. 2012; Yao et al. 2020). In the present study, these four stress-related genes showed significantly elevated expression levels in the transgenic ZmNF-YA12 compared to WT plants under drought and high NaCl treatments (Fig. 7A, B, C, D). These results indicated that ZmNF-YA12 improves salt and drought tolerance by inducing the expression of stress-related genes in Arabidopsis.

Proteins interact with other proteins in complex network systems to perform their diverse and targeted functions (Bhardwaj et al. 2016). A previous study showed that the maize NF-Y family gene NF-YA3 could interact with the JA activator MYC4 to improve drought and heat tolerance (Su et al. 2018). There have been no previous reports regarding the interactions of ZmNF-YA12 proteins in maize. In the present study, yeast two-hybrid experiments showed that ZmNF-YA12 interacted with ZmNF-YC1 and ZmNF-YC15. Our previous study showed that ZmNF-YC15 was induced by drought stress (Zhang et al. 2016). Therefore, ZmNF-YA12 may respond to stress by interacting with ZmNF-YC15.

In conclusion, we cloned and characterized the NF-Y gene ZmNF-YA12 from Zea mays. ZmNF-YA12 was expressed at high levels in young roots and induced by abiotic stresses. Its heterologous expression conferred enhanced tolerance to drought and salt stress by regulating the expression of stress-related genes. The gene may also perform its diverse and targeted functions by interacting with ZmNF-YC1 and ZmNF-YC15. These results will be helpful to understand the roles of NF-Y in abiotic stress responses.

\section{Declarations}

\section{Author contribution statement}

ZZ, RY and ZW conceived and designed the experiments; TZ, DZ and CZ performed the experiments and analyzed the data; $T Z$ wrote the manuscript and $Z Z, R Y$ and $Z W$ revised and approved the publication.

\section{Conflict of interest}

The authors declare that they have no conflicts of interest.

\section{Acknowledgements}


This work was supported by Foundation of Beijing Academy of Agriculture and Forestry Sciences KJCX20200205 and KJCX20200407, National Natural Science Foundation of China 31871351 and 32001430.

The English in this document has been checked by at least two professional editors, both native speakers of English. For a certificate, please see:

\section{http://www.textcheck.com/certificate/V5HJ8f}

\section{References}

1. Agathokleous E, Feng Z, Peñuelas J (2020) Chlorophyll hormesis: Are chlorophylls major components of stress biology in higher plants? Sci Total Environ 726:138637. https://doi.org/10.1016/j.scitotenv.2020.138637

2. Alam MM, Tanaka T, Nakamura H, Ichikawa H, Kobayashi K, Yaeno T, Yamaoka N, Shimomoto K, Takayama K, Nishina H, Nishiguchi M (2015) Overexpression of a rice heme activator protein gene (OsHAP2E) confers resistance to pathogens, salinity and drought, and increases photosynthesis and tiller number. Plant Biotechnol J 13:85-96. https://doi.org/10.1111/pbi.12239

3. Bhardwaj J, Gangwar I, Panzade G, Shankar R, Yadav SK (2016) Global de novo protein-protein interactome elucidates interactions of drought responsive proteins in horsegram (Macrotyloma uniflorum). J Proteome Res 15(6):1794-1809. https://doi.org/10.1021/acs.jproteome.5b01114

4. Deinlein U, Stephan AB, Horie T, Luo W, Xu G, Schroeder JI (2014) Plant salt-tolerance mechanisms. Trends Plant Sci 19:371-379. https://doi.org/10.1016/j.tplants.2014.02.001

5. Lee DK, Kim HI, Jang G, Chung PJ, Jeong JS, Kim YS, Bang SW, Jung H, Choi YD, Kim JK (2015) The NF-YA transcription factor OsNF-YA7 confers drought stress tolerance of rice in an abscisic acid independent manner. Plant Science 241:199-210. https://doi.org/10.1016/j.plantsci.2015.10.006

6. Feng ZJ, He GH, Zheng WJ, Lu PP, Chen M, Gong YM, Ma YZ, Xu ZS (2015) Foxtail millet NF-Y families: genome-wide survey and evolution analyses identified two functional genes important in abiotic stresses. Front Plant Sci 6:1142. https://doi.org/10.3389/fpls.2015.01142

7. Gong F, Yang L, Tai F, Hu X, Wang W (2014) "Omics" of maize stress response for sustainable food production: opportunities and challenges. OMICS 18:714-

732. https://doi.org/10.1089/omi.2014.0125

8. Kumimoto RW, Adam L, Hymus GJ, Repetti PP, Reuber TL, Marion CM, Hempel FD, Ratcliffe OJ (2008) The Nuclear Factor Y subunits NF-YB2 and NF-YB3 play additive roles in the promotion of flowering by inductive long-day photoperiods in Arabidopsis. Planta 228:709723. https://doi.org/10.1007/s00425-008-0773-6

9. Lehmann S, Funck D, Szabados L, Rentsch D (2010) Proline metabolism and transport in plant development. Amino Acids 39:949-962. https://doi.org/10.1007/s00726-010-0525-3 
10. Liebthal M, Maynard D, Dietz KJ (2018) Peroxiredoxins and redox signaling in plants. Antioxid Redox Signal 28:609-624. https://doi.org/10.1089/ars.2017.7164

11. Ma X, Li C, Wang M (2015) Wheat NF-YA10 functions independently in salinity and drought stress. Bioengineered 6:245-247. https://doi.org/10.1080/21655979.2015.1054085

12. Maity SN, de Crombrugghe B (1998) Role of the CCAAT-binding protein CBF/NF-Y in transcription. Trends Biochem Sci 23:174-178. https://doi.org/10.1016/s0968-0004(98)01201-8

13. Msanne J, Lin J, Stone JM, Awada T (2011) Characterization of abiotic stress-responsive Arabidopsis thaliana RD29A and RD29B genes and evaluation of transgenes. Planta 234:97107. https://doi.org/10.1007/s00425-011-1387-y

14. Nakashima K, Fujita Y, Katsura K, Maruyama K, Narusaka Y, Seki M, Shinozaki K (2006) Transcriptional regulation of ABI3- and ABA-responsive genes including RD29B and RD29A in seeds, germinating embryos, and seedlings of Arabidopsis. Plant Mol Biol 60:5168. https://doi.org/10.1007/s11103-005-2418-5

15. Nowicka B, Ciura J, Szymańska R, Kruk J (2018) Improving photosynthesis, plant productivity and abiotic stress tolerance - current trends and future perspectives. J Plant Physiol 231:415433. https://doi.org/10.1016/j.jplph.2018.10.022

16. Palmeros-Suárez PA, Massange-Sánchez JA, Martínez-Gallardo NA, Montero-Vargas JM, GómezLeyva JF, Délano-Frier JP (2015) The overexpression of an Amaranthus hypochondriacus NF-YC gene modifies growth and confers water deficit stress resistance in Arabidopsis. Plant Sci 240:2540. https://doi.org/10.1016/j.plantsci.2015.08.010

17. Pechanova O, Takáč T, Samaj J, Pechan T (2013) Maize proteomics: an insight into the biology of an important cereal crop. Proteomics 13:637-662. https://doi.org/10.1002/pmic.201200275

18. Pereira SLS, Martins CPS, Sousa AO, Camillo LR, Araújo CP, Alcantara GM, Camargo DS, Cidade LC, de Almeida AF, Costa MGC (2018) Genome-wide characterization and expression analysis of citrus NUCLEAR FACTOR-Y (NF-Y) transcription factors identified a novel NF-YA gene involved in droughtstress response and tolerance. PLoS One 13: e0199187. https://doi.org/10.1371/journal.pone.0199187

19. Petroni K, Kumimoto RW, Gnesutta N, Calvenzani V, Fornari M, Tonelli C, Holt BF, Mantovani R (2012) The promiscuous life of plant NUCLEAR FACTOR Y transcription factors. Plant Cell 24:47774792. https://doi.org/10.1105/tpc.112.105734

20. Romier C, Cocchiarella F, Mantovani R, Moras D (2003) The NF-YB/NF-YC structure gives insight into DNA binding and transcription regulation by CCAAT factor NF-Y. J Biol Chem 278:13361345. https://doi.org/10.1074/jbc.M209635200

21. Rushton DL, Tripathi P, Rabara RC, Lin J, Ringler P, Boken AK, Langum TJ, Smidt L, Boomsma DD, Emme NJ, Chen X, Finer JJ, Shen QJ, Rushton PJ (2012) WRKY transcription factors: key components in abscisic acid signalling. Plant Biotechnol J 10:2-11. https://doi.org/10.1111/j.14677652.2011.00634.x 
22. Su H, Cao Y, Ku L, Yao W, Cao Y, Ren Z, Dou D, Wang H, Ren Z, Liu H, Tian L, Zheng Y, Chen C, Chen Y (2018) Dual functions of ZmNF-YA3 in photoperiod-dependent flowering and abiotic stress responses in maize. J Exp Bot 69:5177-5189. https://doi.org/10.1093/jxb/ery299

23. Tiburcio AF, Wollenweber B, Zilberstein A, Koncz C (2012) Abiotic stress tolerance. Plant Science 182:1-2. https://doi.org/10.1016/j.plantsci.2011.09.005

24. Tsikas $D$ (2017) Assessment of lipid peroxidation by measuring malondialdehyde (MDA) and relatives in biological samples: Analytical and biological challenges. Anal Biochem 524:13-30. https://doi.org/10.1016/j.ab.2016.10.021

25. Yamaguchi-Shinozaki K, Shinozaki K (2006) Transcriptional regulatory networks in cellular responses and tolerance to dehydration and cold stresses. Annu Rev Plant Biol 57:781803. https://doi.org/10.1146/annurev.arplant.57.032905.105444

26. Yao L, Hao X, Cao H, Ding C, Yang Y, Wang L, Wang X (2020) ABA-dependent bZIP transcription factor, CsbZIP18, from Camellia sinensis negatively regulates freezing tolerance in Arabidopsis. Plant Cell Rep 39:553-565. https://doi.org/10.1007/s00299-020-02512-4

27. Zanetti ME, Rípodas C, Niebel A (2017) Plant NF-Y transcription factors: Key players in plant-microbe interactions, root development and adaptation to stress. Biochim Biophys Acta Gene Regul Mech 1860:645-654. https://doi.org/10.1016/j.bbagrm.2016.11.007

28. Zhang Z, Li X, Zhang C, Zou H, Wu Z (2016) Isolation, structural analysis, and expression characteristics of the maize nuclear factor $Y$ gene families. Biochem Biophys Res Commun 478:752758. https://doi.org/10.1016/j.bbrc.2016.08.020

29. Zhou Y, Zhang Y, Wang X, Han X, An Y, Lin S, Shen C, Wen J, Liu C, Yin W, Xia X (2020) Root-specific NF-Y family transcription factor, PdNF-YB21, positively regulates root growth and drought resistance by abscisic acid-mediated indoylacetic acid transport in Populus. New Phytol 227:407-426. https://doi.org/10.1111/nph.16524

\section{Figures}


A

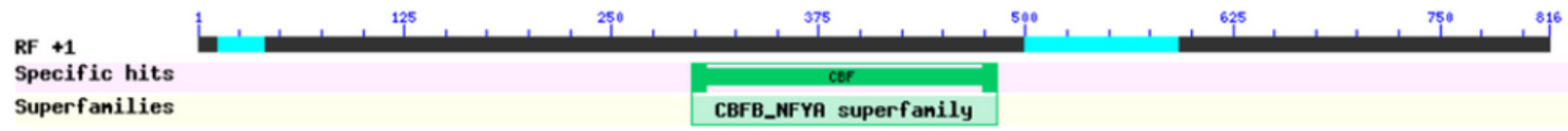

B

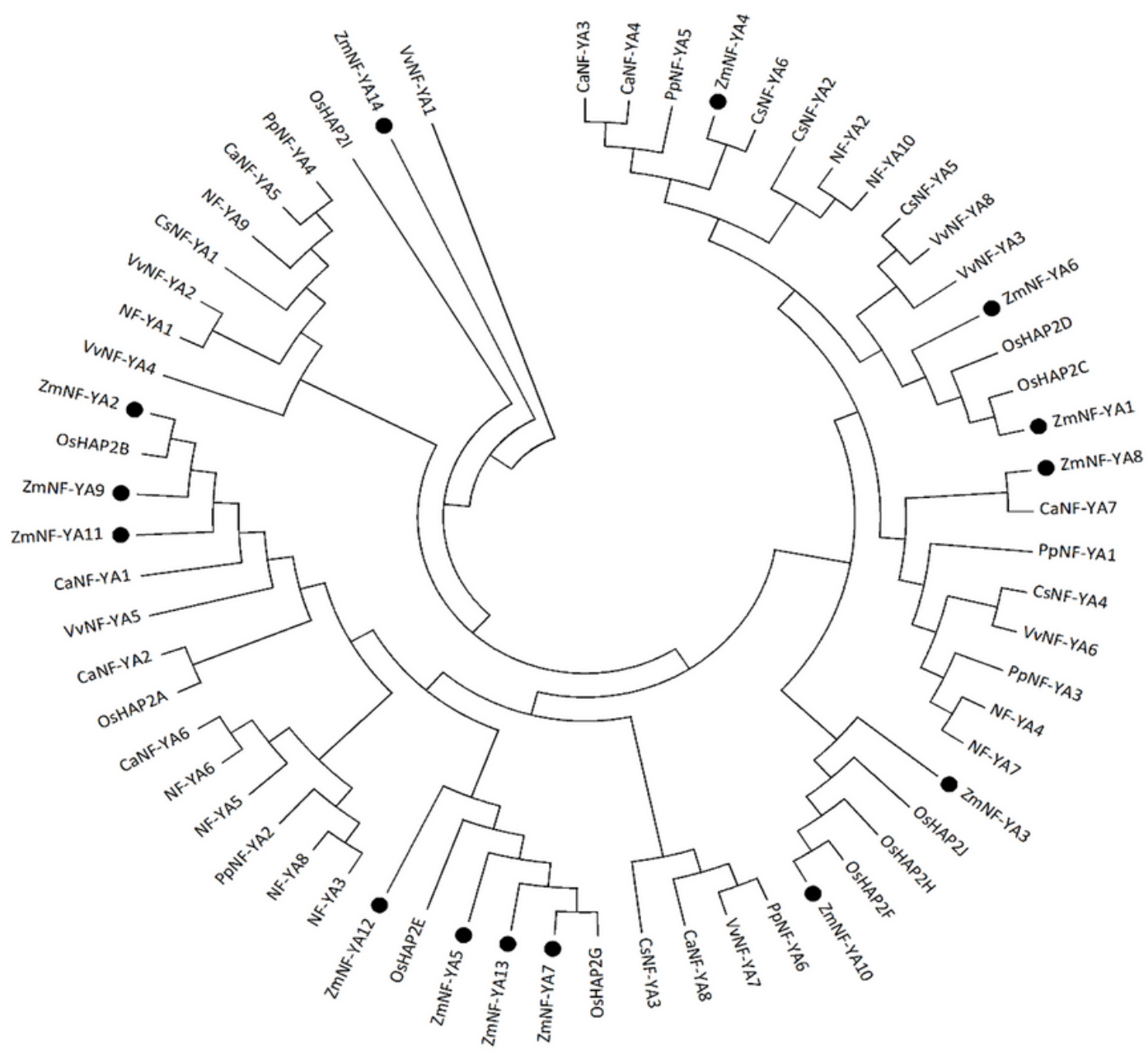

Figure 1

Bioinformatics analysis of the maize ZmNF-YA12 protein. A. Analysis of the ZmNF-YA12 conserved domain. B. Phylogenetic relationships of NF-YA family members in maize and other plants. All of the NFYA family sequences were obtained from the NCBI database. 


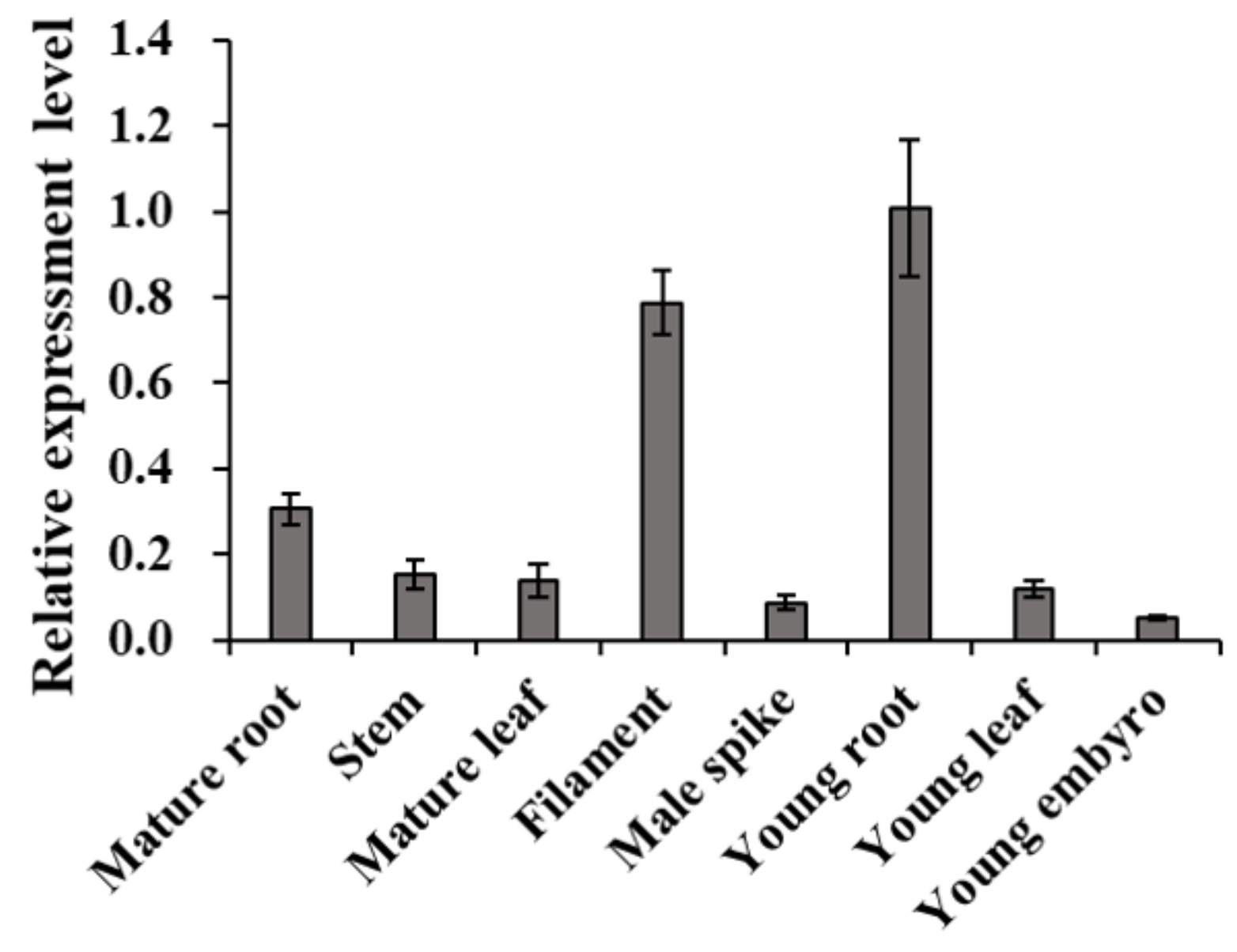

Figure 2

Tissue expression pattern of ZmNF-YA12 in maize. Total RNA was extracted from various tissues. GAPDH was used as an internal control. The vertical column shows the relative transcript level. Data are shown as the mean $\pm \operatorname{SEM}(n=3)$. 
A

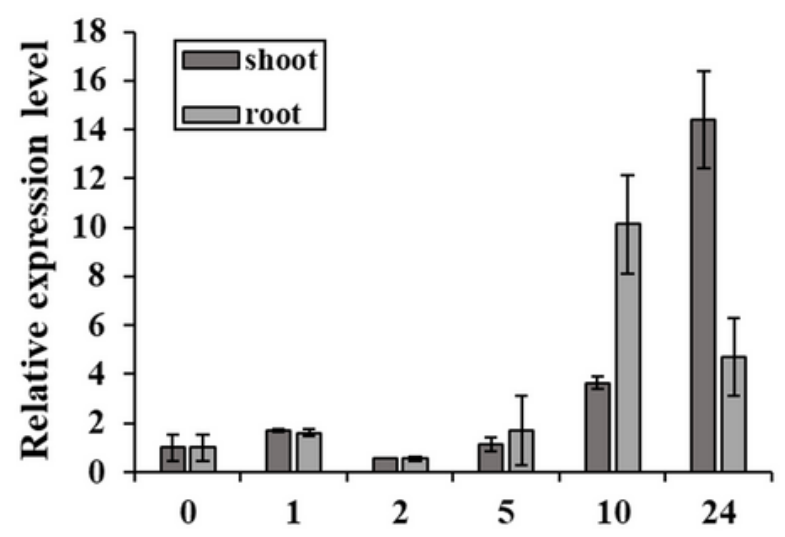

Dehydration treatment time(h)

C

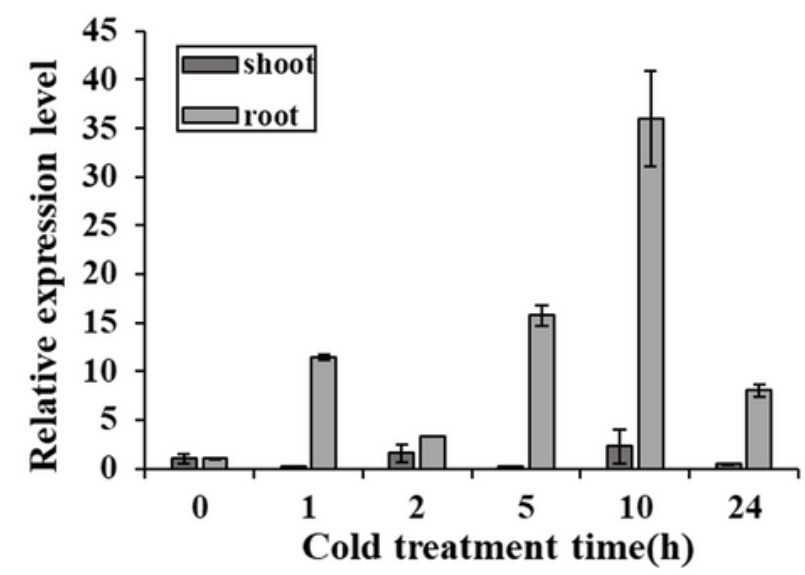

$\mathbf{E}$

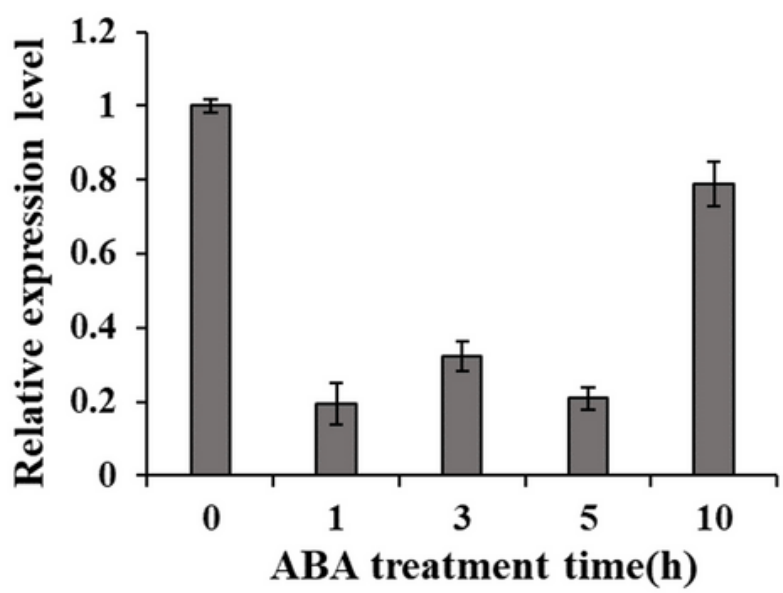

B

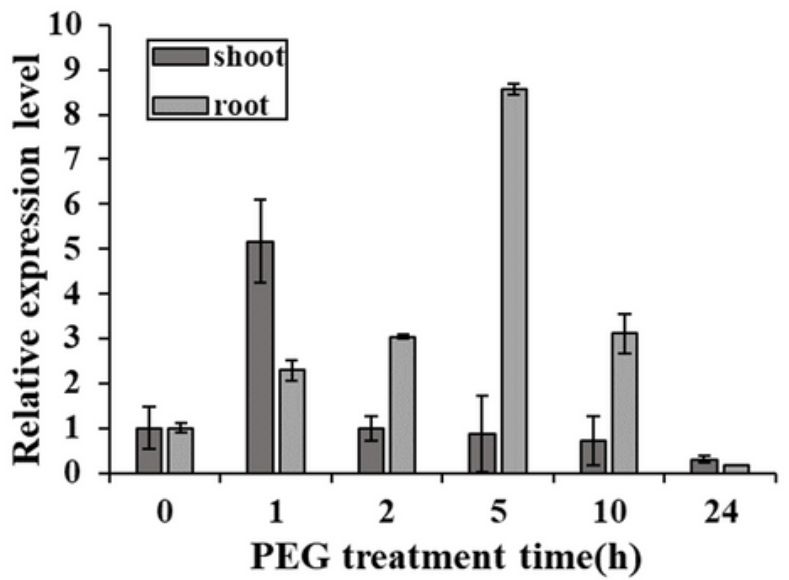

D

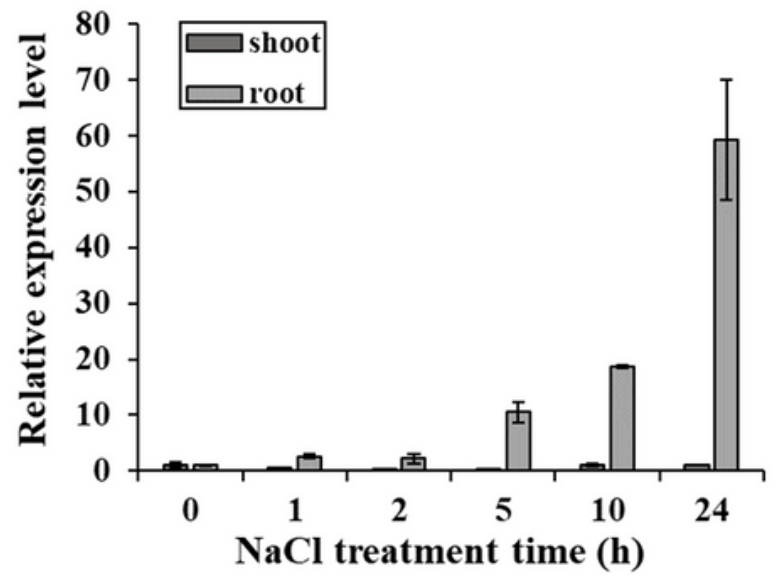

$\mathbf{F}$

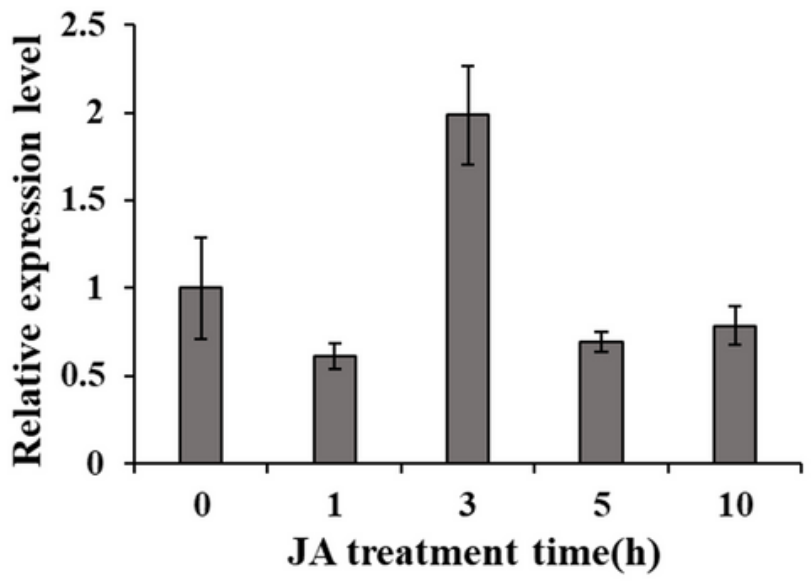

Figure 3

Expression of ZmNF-YA12 in response to abiotic and hormone stress in maize. A-F. ZmNF-YA12 expression under conditions of dehydration, PEG, cold, $\mathrm{NaCl}, \mathrm{ABA}$, and JA stress. GAPDH was used as an internal control. Data are shown as the mean \pm SEM $(n=3)$. 
A
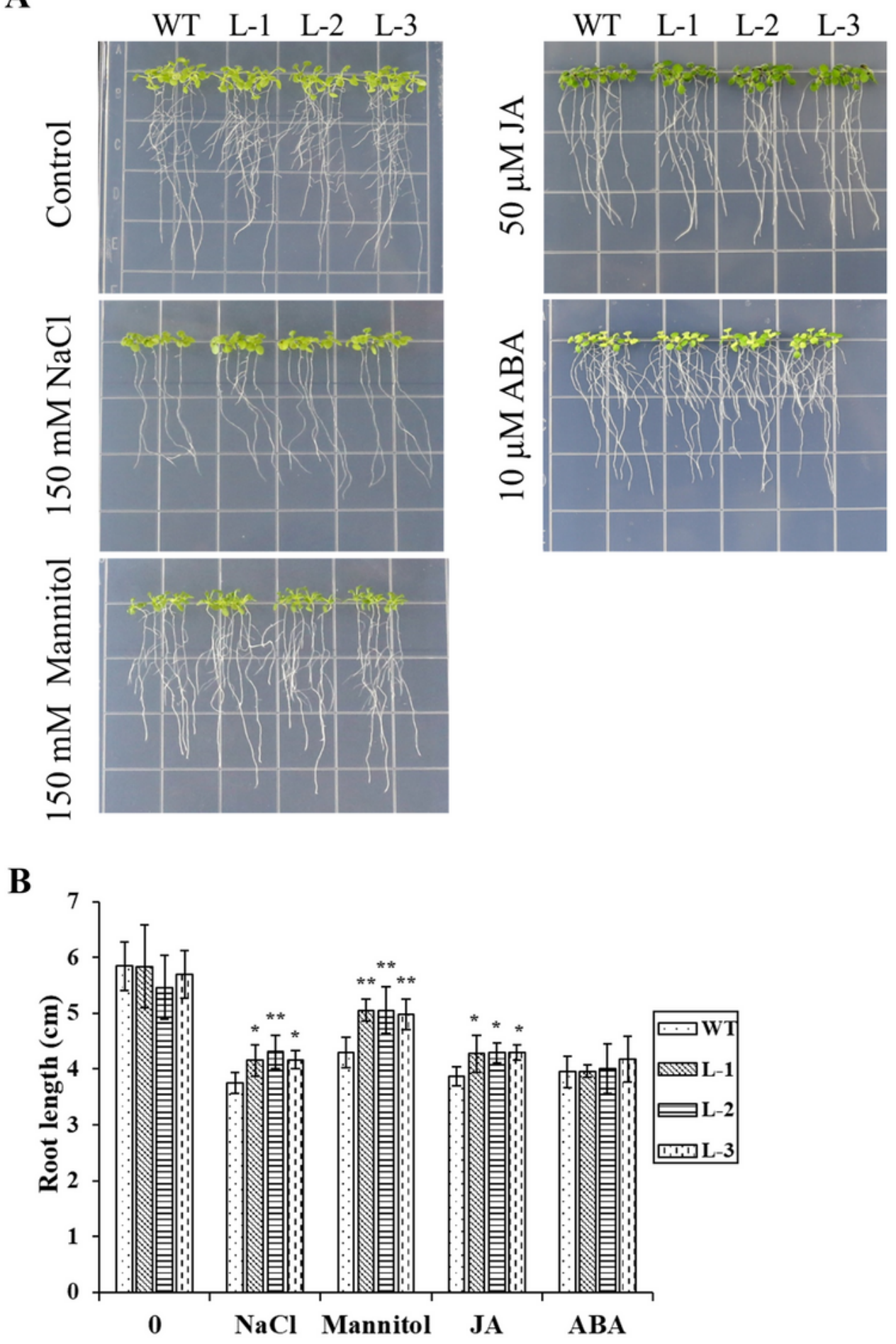

\section{Figure 4}

Phenotype analysis of WT and transgenic Arabidopsis lines under $\mathrm{NaCl}$, mannitol, JA, and ABA treatments. A. Seedling development of transgenic and WT plants on $1 / 2 \mathrm{MS}$ medium or $1 / 2 \mathrm{MS}$ supplemented with $150 \mathrm{mM} \mathrm{NaCl}, 150 \mathrm{mM}$ mannitol, $50 \mu \mathrm{M} \mathrm{JA}$, or $10 \mu \mathrm{M} \mathrm{ABA}$. B. Primary root length of plants grown on different media. Data represent the average root lengths. ${ }^{*} p<0.05 ;{ }^{*} p<0.01$ compared to the corresponding controls. Bar $=1.5 \mathrm{~cm}$. 
A
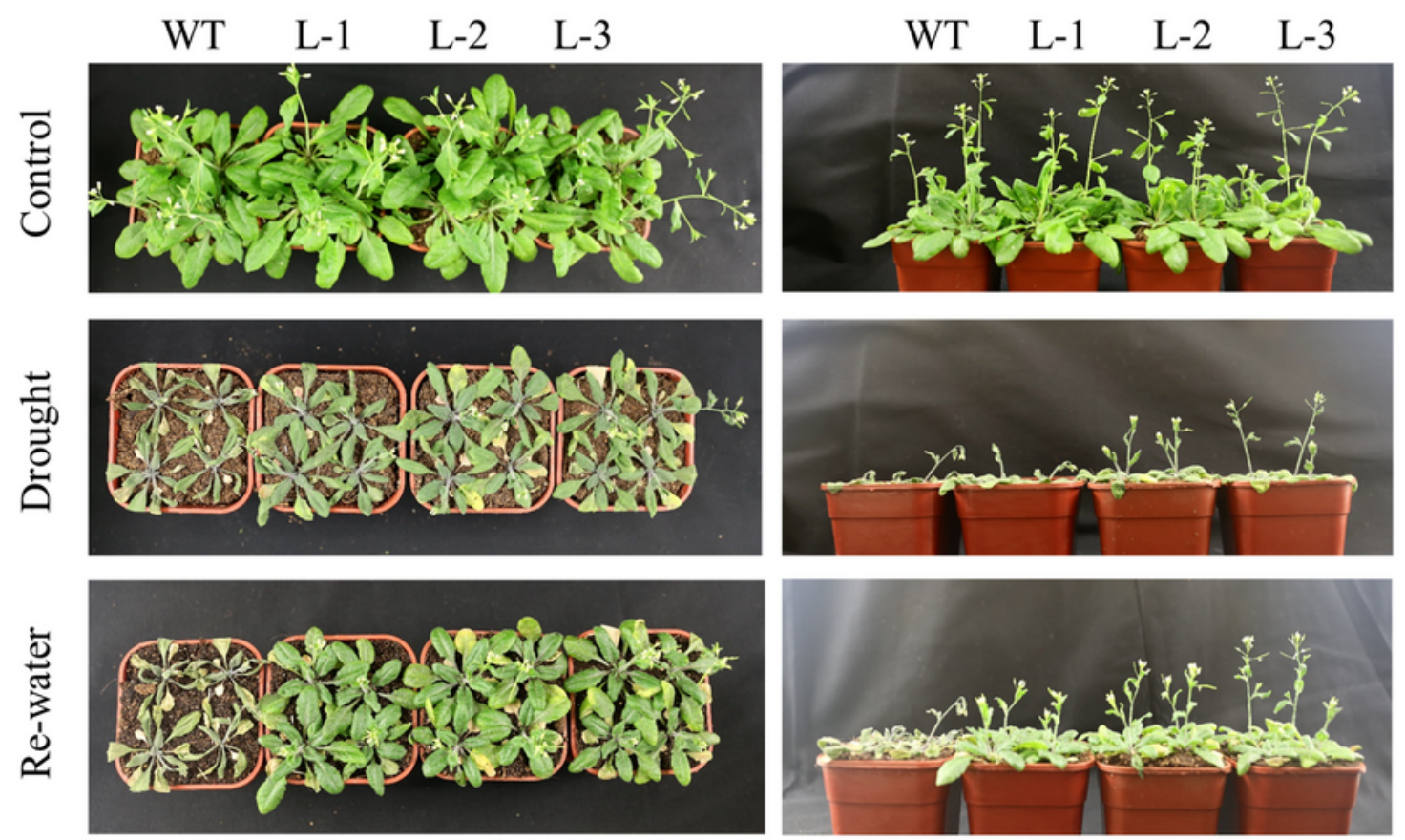

B

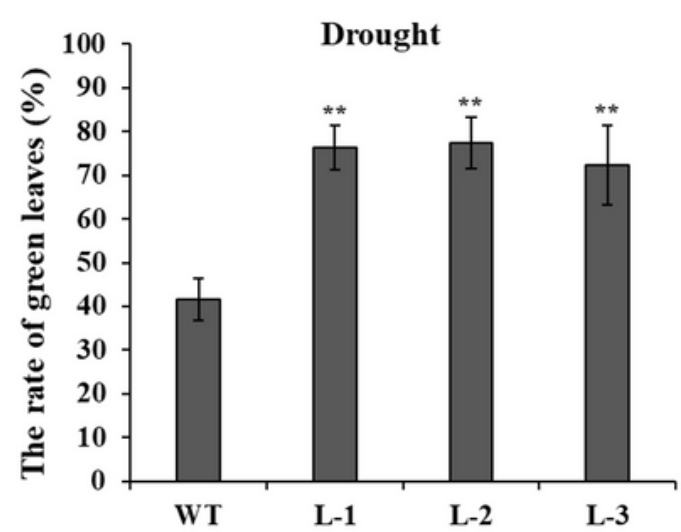

C

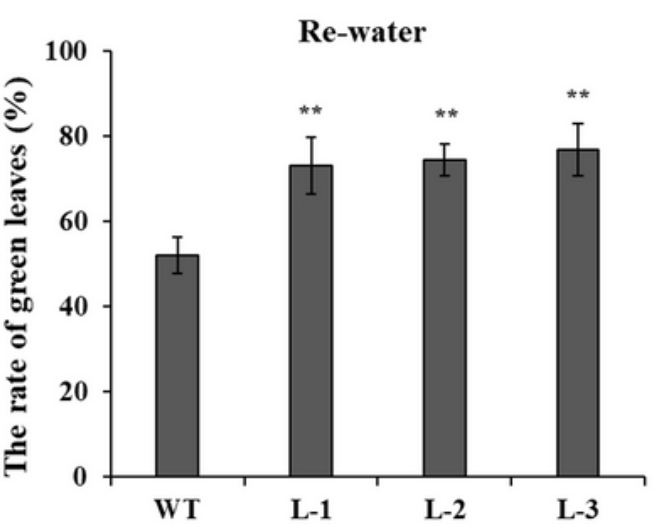

D

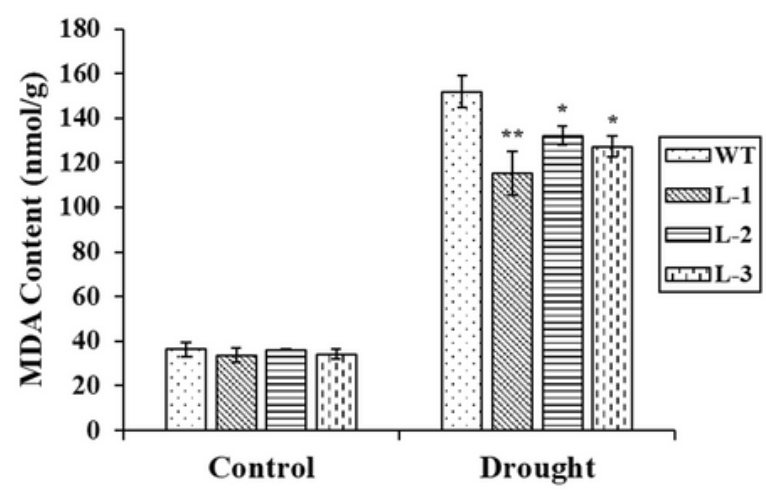

$\mathbf{E}$

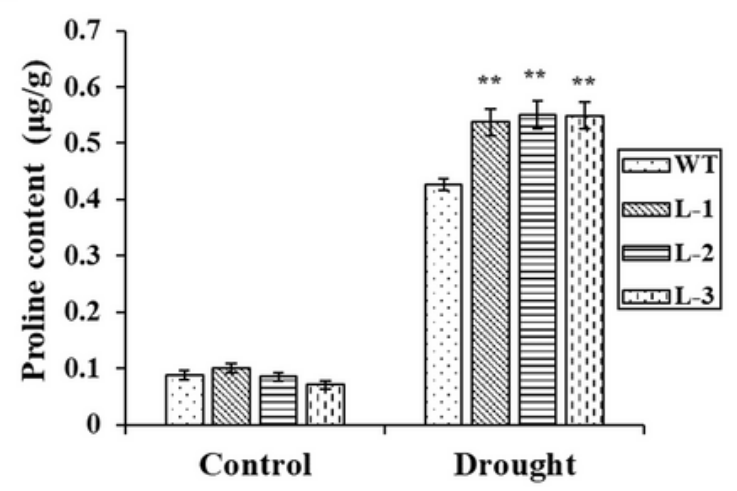

Figure 5

Improved drought tolerance in transgenic Arabidopsis plants expressing ZmNF-YA12. A. Assessment of drought tolerance in transgenic Arabidopsis plants. Healthy WT and 35S:ZmNF-YA12 plants were grown for 20 days with or without (control) water deficit, followed by re-watering for 2 days. B, C. The rates of green leaves in WT and transgenic lines under drought and re-watering treatments. D, E. Measurements of MDA and proline contents in transgenic lines and WT plants. ${ }^{*} p<0.05 ;{ }^{* \star} p<0.01$. 
A
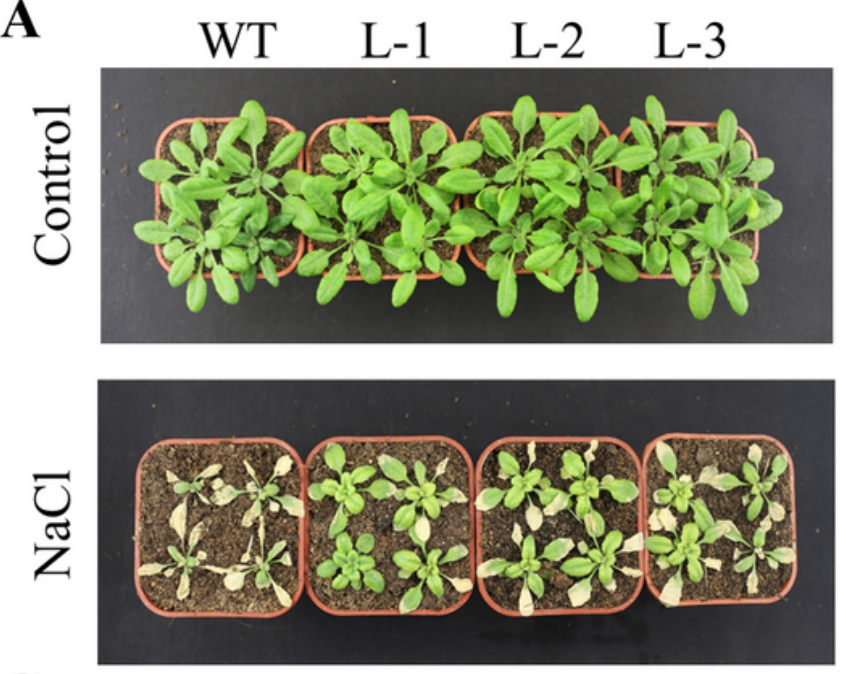

C

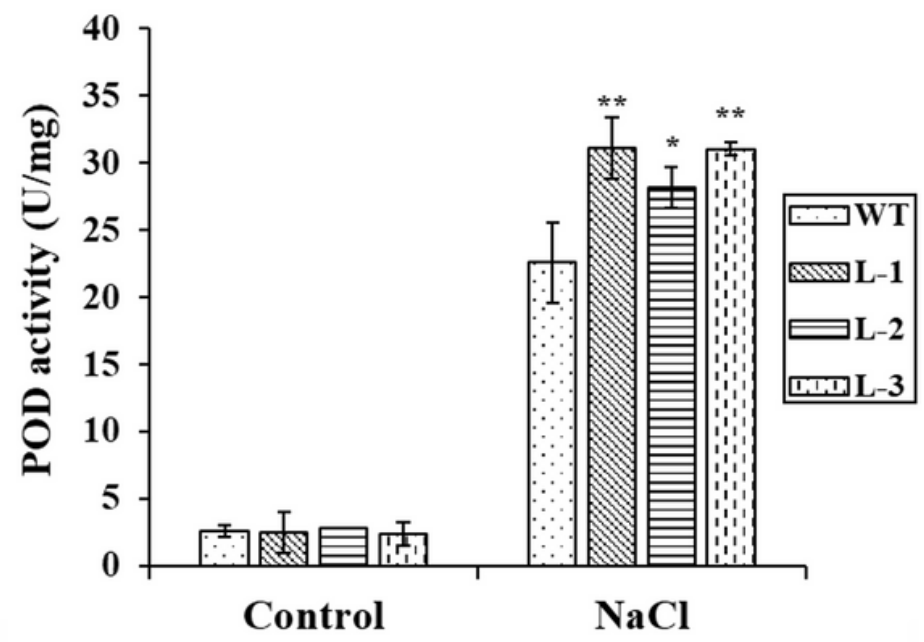

B

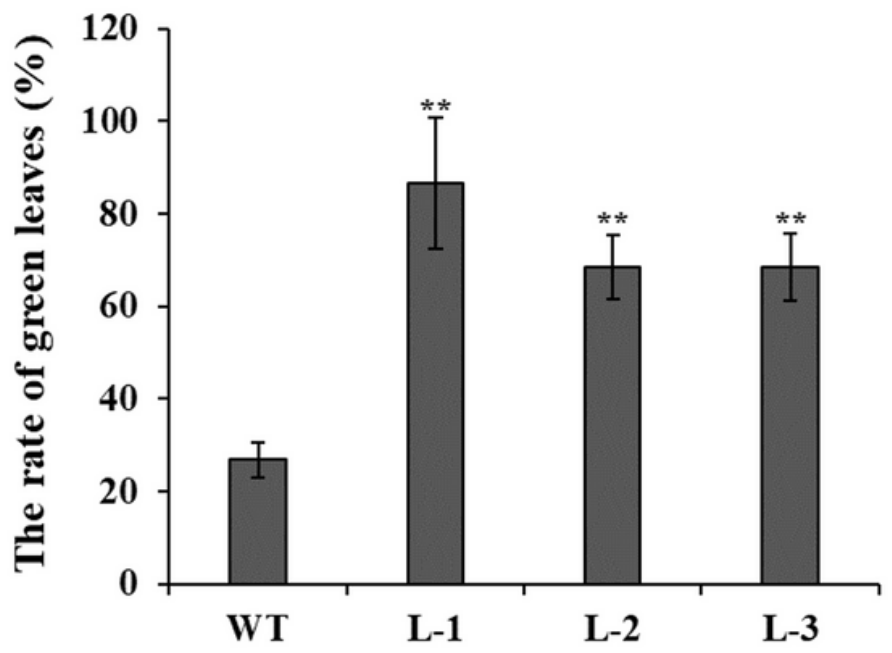

D

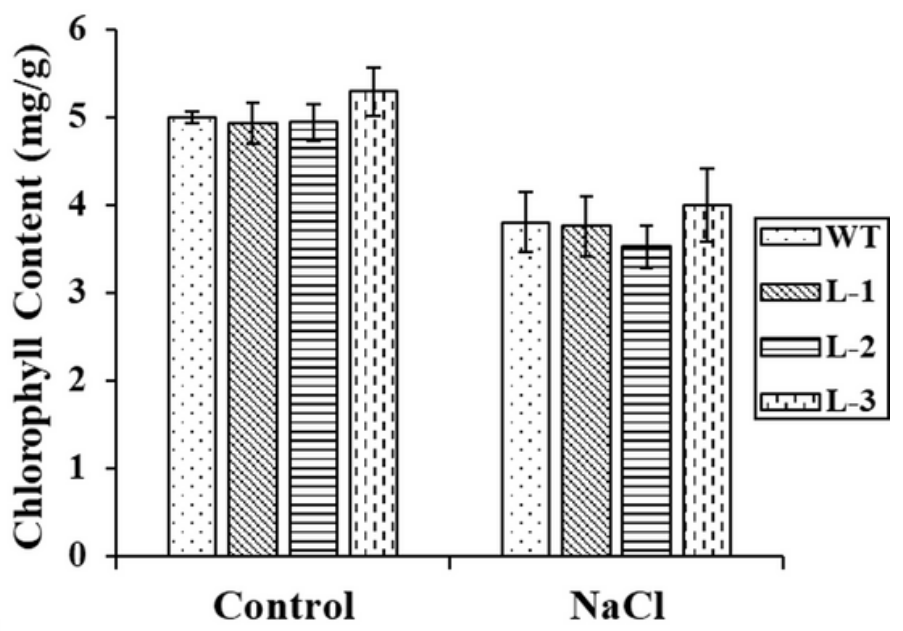

Figure 6

Enhanced salt tolerance of transgenic plants expressing ZmNF-YA12. A. Phenotypes of WT and transgenic seedlings in soil with or without (control) $450 \mathrm{mM} \mathrm{NaCl}$ treatment. Four-week-old transgenic and WT plants were irrigated with $\mathrm{NaCl}$ solution. Photographs were taken 7 days after treatment. B. Statistical analysis of green leaves of 35S:ZmNF-YA12 and WT plants. C, D. POD activity and chlorophyll content were measured in transgenic and WT plants. ${ }^{*} p<0.05$; ${ }^{*} p<0.01$ compared to the corresponding controls. 
A

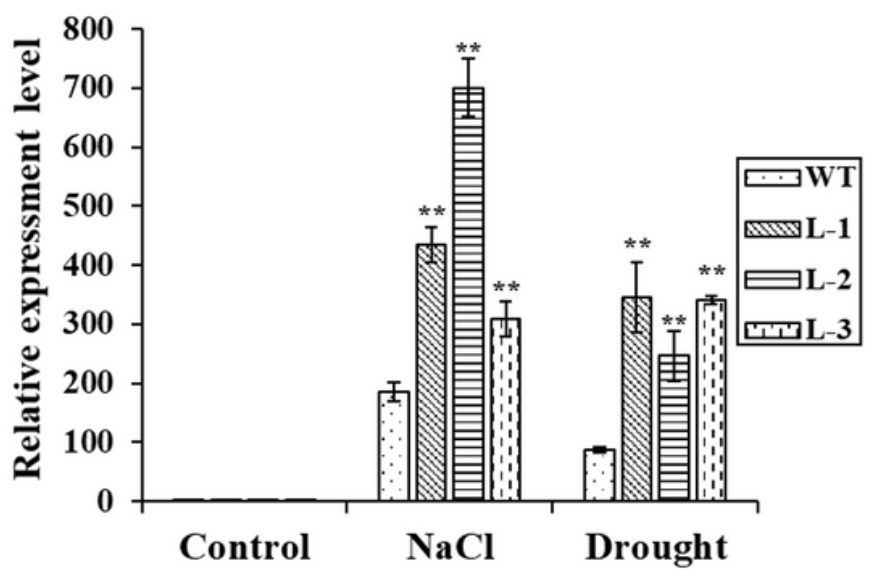

C

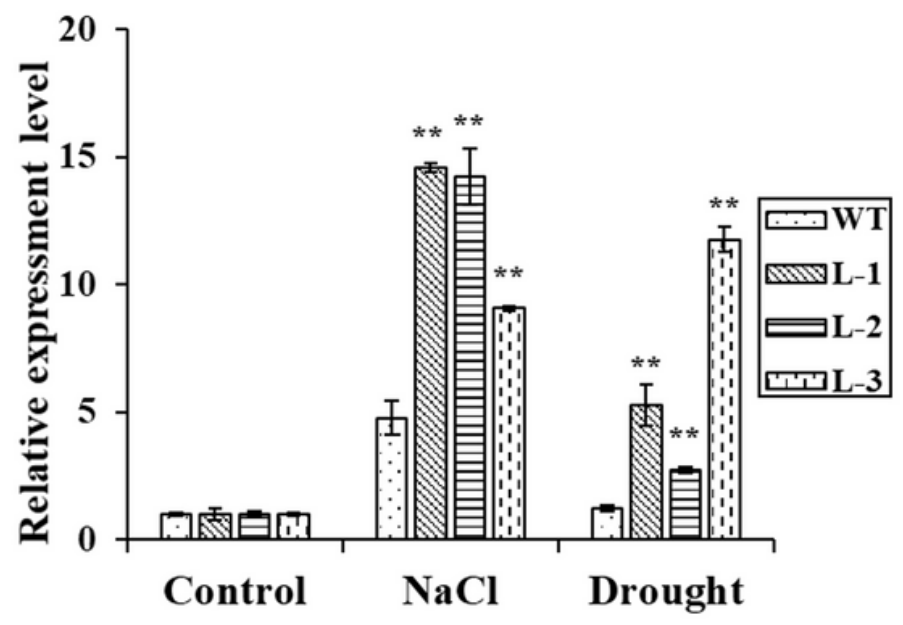

B

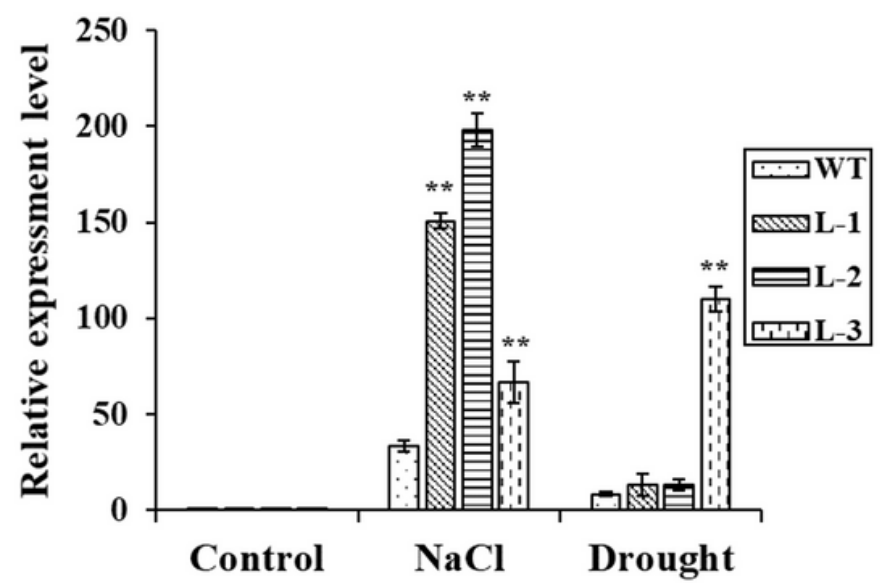

D

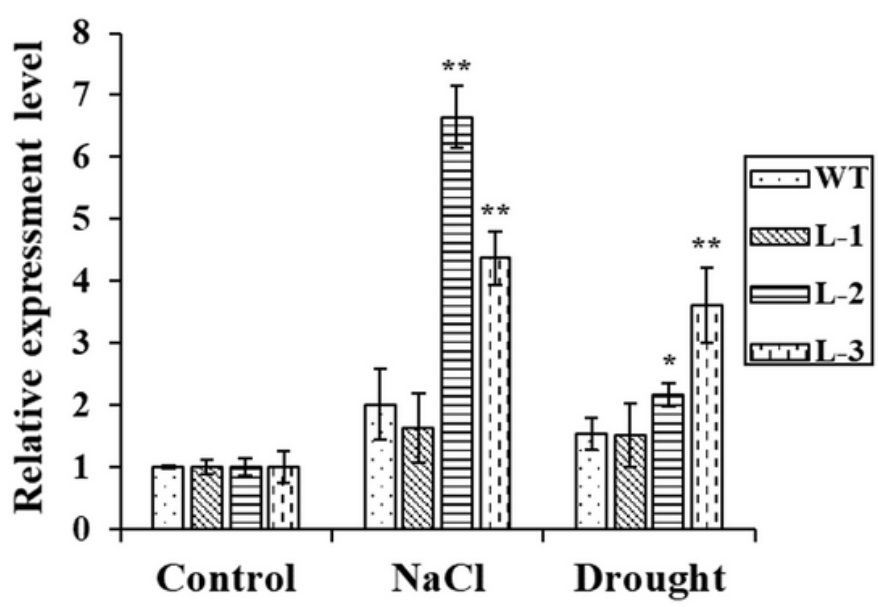

Figure 7

RD29A, RD29B, RAB18, and RD22 transcript levels in WT and ZmNF-YA12 transgenic plants under three growth conditions. Actin2 was used as an internal control. Data represent the mean $\pm \operatorname{SEM}(n=3) .{ }^{*} p<$ $0.05 ; * \star p<0.01$. 


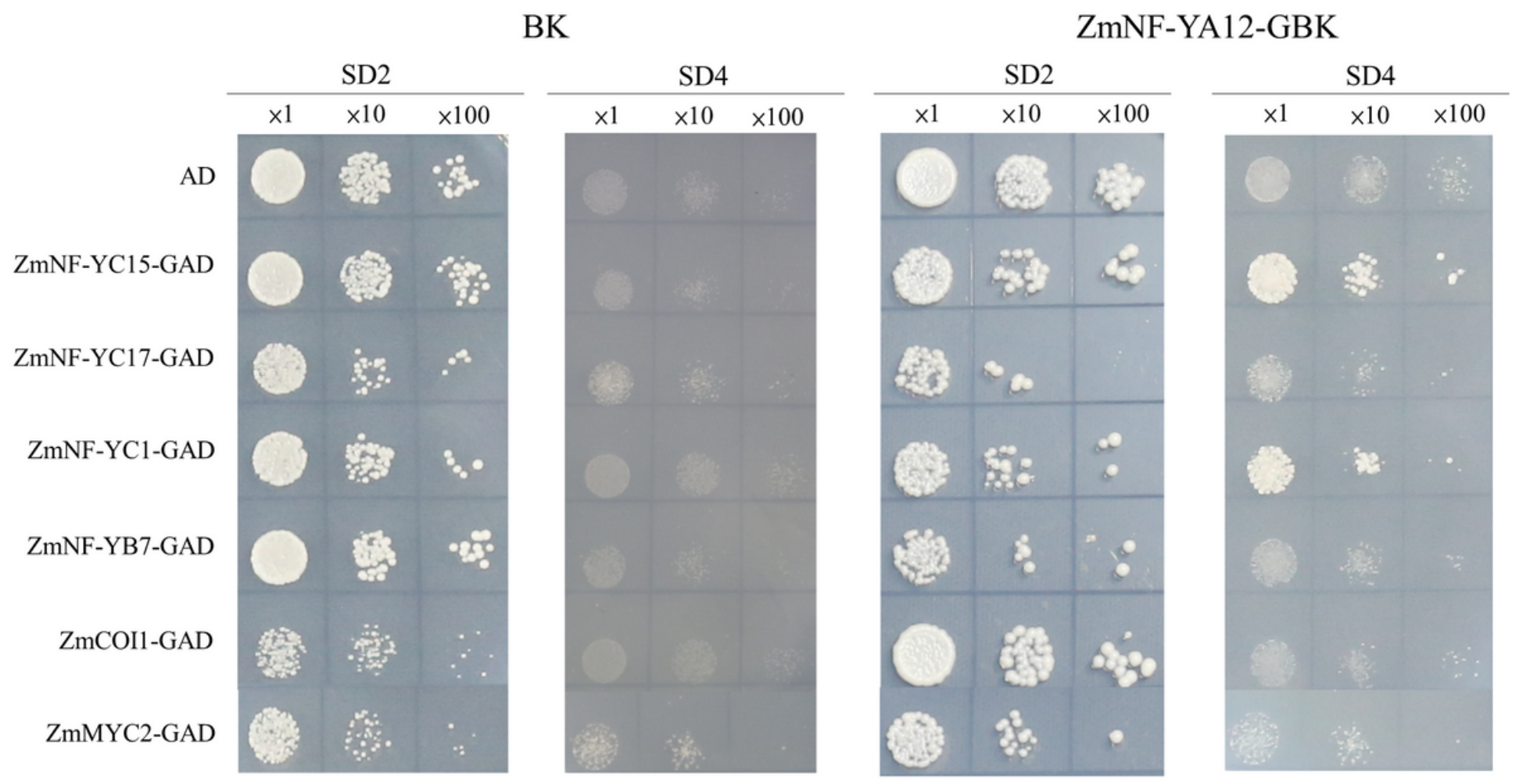

Figure 8

Protein interaction assays between ZmNF-YA12 and candidate genes.

\section{Supplementary Files}

This is a list of supplementary files associated with this preprint. Click to download.

- SupplementaryFigures.docx 